\title{
PASSO-A-PASSO PARA UTILIZAÇÃO DE FIBRINA RICA EM PLAQUETAS COMO AGENTE HEMOSTÁTICO APÓS EXODONTIA : DESCRIÇÃO DA TÉCNICA
}

Step-by-step for use of platelet-rich fibrin as a hemostatic agent after exodontic: description of the technique

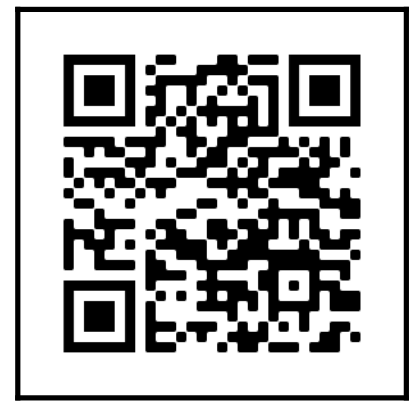

\section{Autores:}

\section{Amanda Vidal de Sousa}

Graduada em Odontologia pela Faculdade de Odontologia da Universidade Federal Fluminense, Rio de Janeiro, RJ, Brasil.

\section{Elisa Siqueira Mendes}

Graduanda em Odontologia pela Faculdade de Odontologia da Universidade Federal Fluminense, Rio de Janeiro, RJ, Brasil.

\section{Leticia Gonçalves Ferreira}

Graduanda em Odontologia pela Faculdade de Odontologia da Universidade Federal Fluminense, Rio de Janeiro, RJ, Brasil.

\section{Fernanda Britto de Melo}

Graduada em Odontologia pela Faculdade de Odontologia da Universidade Federal Fluminense, Rio de Janeiro, RJ, Brasil

Especialista em Cirurgia e Traumatologia Buco-Maxilo-Facial pela Universidade Federal Fluminense, Rio de Janeiro, RJ, Brasil.

\section{Carlos Fernando de Almeida Barros Mourão}

Doutor em Odontologia pela Faculdade de Odontologia da Universidade Federal Fluminense, Rio de Janeiro, RJ, Brasil.

\section{Mônica Diuana Calasans-Maia}

Professora das disciplinas de Cirurgia Oral Menor e Anestesiologia do curso de Odontologia da Universidade Federal Fluminense, Niterói, RJ, Brasil.

\section{Suelen Cristina Sartoretto}


Professora das disciplinas de Cirurgia Bucal I, II e III da Universidade Iguaçu, Nova Iguaçu, RJ, Brasil. E Professora dos cursos de mestrado e doutorado em Odontologia da Universidade Veiga de Almeida, RJ, Brasil.

\section{Marcelo José Uzeda}

Professor das disciplinas de Cirurgia Bucal I, II e III da Universidade Iguaçu, Nova Iguaçu, RJ, Brasil.

Professor das disciplinas de Cirurgia Oral Menor e Anestesiologia do curso de Odontologia da Universidade Federal Fluminense, Niterói, RJ, Brasil.

\section{Rodrigo Figueiredo de Brito Resende}

Professor das disciplinas de Cirurgia Bucal I, II e III da Universidade Iguaçu, Nova Iguaçu, RJ, Brasil.

Professor das disciplinas de Cirurgia Oral Menor e Anestesiologia do curso de Odontologia da Universidade Federal Fluminense, Niterói, RJ, Brasil.

Instituição na qual o trabalho foi realizado: Universidade Federal Fluminense, Niterói, RJ, Brasil.

\section{Endereço para correspondência:}

Fernanda Britto de Melo Silva

Rua Mário Santos Braga, 28 - Centro, Niterói - RJ, 24020-140

Telefone: (21) 99817-7665

E-mail: fernandabrittodemelo2@hotmail.com

\section{RESUMO}

A extração dentária é um dos procedimentos mais frequentes em cirurgia oral e maxilofacial e está relacionada a mudanças fisiológicas no processo alveolar. Neste sentido, entre as principais complicações transoperatórias está a hemorragia, que ocorre geralmente devido a lesões de vasos sanguíneos presentes no alvéolo dentário onde se realizou a exodontia. Uma alternativa para se obter a hemostasia é a utilização da Fibrina Rica em Plaquetas (PRF), um concentrado leucoplaquetário, obtido através do sangue do paciente. A membrana obtida é rica em leucócitos, plaquetas e fatores de crescimento que promovem a modulação de células envolvidas no processo de cicatrização, favorecendo um melhor e mais rápido reparo das lesões cirúrgicas. Esta matriz de fibrina apresenta diversas utilidades para a odontologia, demonstrando bons resultados, além do baixo custo e fácil obtenção. O objetivo deste artigo é 
descrever a técnica de obtenção da PRF usada como agente hemostático após exodontia, orientando assim, sua reprodutibilidade e utilização.

Palavras-chave: Fibrina Rica em Plaquetas; hemostático; odontologia; exodontia.

\section{ABSTRACT}

Tooth extraction is one of the most frequent procedures in oral and maxillofacial surgery and is related to physiological changes in the alveolar process. In this sense, among the main transoperative complications is hemorrhage, which usually occurs through the dental alveolus, due to damage to the blood vessels where the extraction was performed. An alternative to obtain hemostasis is the use of Fibrin Rich in Platelets (PRF), which is a white platelet concentrate obtained from the patient's blood that undergoes a centrifugation step. The membrane obtained after this process is rich in leukocytes, platelets and growth factors that promote modulation of cells involved in the healing process, favoring a better and faster repair of surgical lesions. This fibrin matrix has several uses for dentistry, showing good results, in addition to being low cost and easy to obtain. The aim of this article is to describe the technique for obtaining Platelet-Rich Fibrin (PRF) used as a hemostatic after tooth socket extraction, thus allowing its reproducibility and use.

Keywords: Platelet-rich fibrin; hemostatic; dentistry; extraction.

\section{INTRODUÇÃO}

O cirurgião buco-maxilo-facial se depara, na sua rotina de trabalho, com diversas cirurgias, sendo mais comum as exodontias . A cicatrização fisiológica do alvéolo pós-extração envolve complexos processos de migração e maturação de células ósseas que levam à reabsorção e consequente perda de volume desse tecido na região1-3. O sangramento é uma das principais intercorrências durante os procedimentos cirúrgicos orais. $\mathrm{Na}$ maioria dos casos, é ocasionado por lesão de vasos sanguíneos da região operada, acontecendo via alvéolo dental4. Nesse sentido, a PRF é uma boa opção como hemostático após exodontia, sendo um produto autólogo, de baixo custo e com produção rápidade aproximadamente 10 minutos. É um biomaterial que 
promove um bom tamponamento mecânico, auxilia na formação do coágulo e favorece a cicatrização tecidual4, liberando de forma contínua no local uma ampla gama de fatores de crescimento6. Além disso, a PRF auxilia na melhora da profundidade de sondagem, na densidade óssea, reduz o inchaço e o trismo7

\begin{tabular}{|l|}
\hline \multicolumn{1}{|c|}{ Vantagens do uso da PRF como hemostático } \\
\hline - Utiliza apenas o sangue autólogo; \\
\hline Dentre os tipos de concentrados plaquetários, é o mais semelhante ao coágulo natural; \\
\hline - Diminui a morbidade de procedimentos cirúrgicos, devido ao seu alto poder de hemostasia; \\
\hline Efíciente no combate aos sinais cardinais da inflamação, como dor e edema, através da modulação da \\
resposta inflamatória local; \\
\hline Tem boa aceitação por parte dos pacientes; \\
\hline Pode ser obtido por meio de uma única etapa de centrifugação; \\
\hline $\begin{array}{l}\text { Podem ser usados após a exodontia, a fim de melhorar a cicatrização do tecido mole, a profundidade de } \\
\text { sondagem, além de auxiliar na redução do trismo e do edema pós-operatório; } \\
\text { - Possui baixo custo. }\end{array}$ \\
\hline
\end{tabular}

\section{GUIA PRÁTICO}
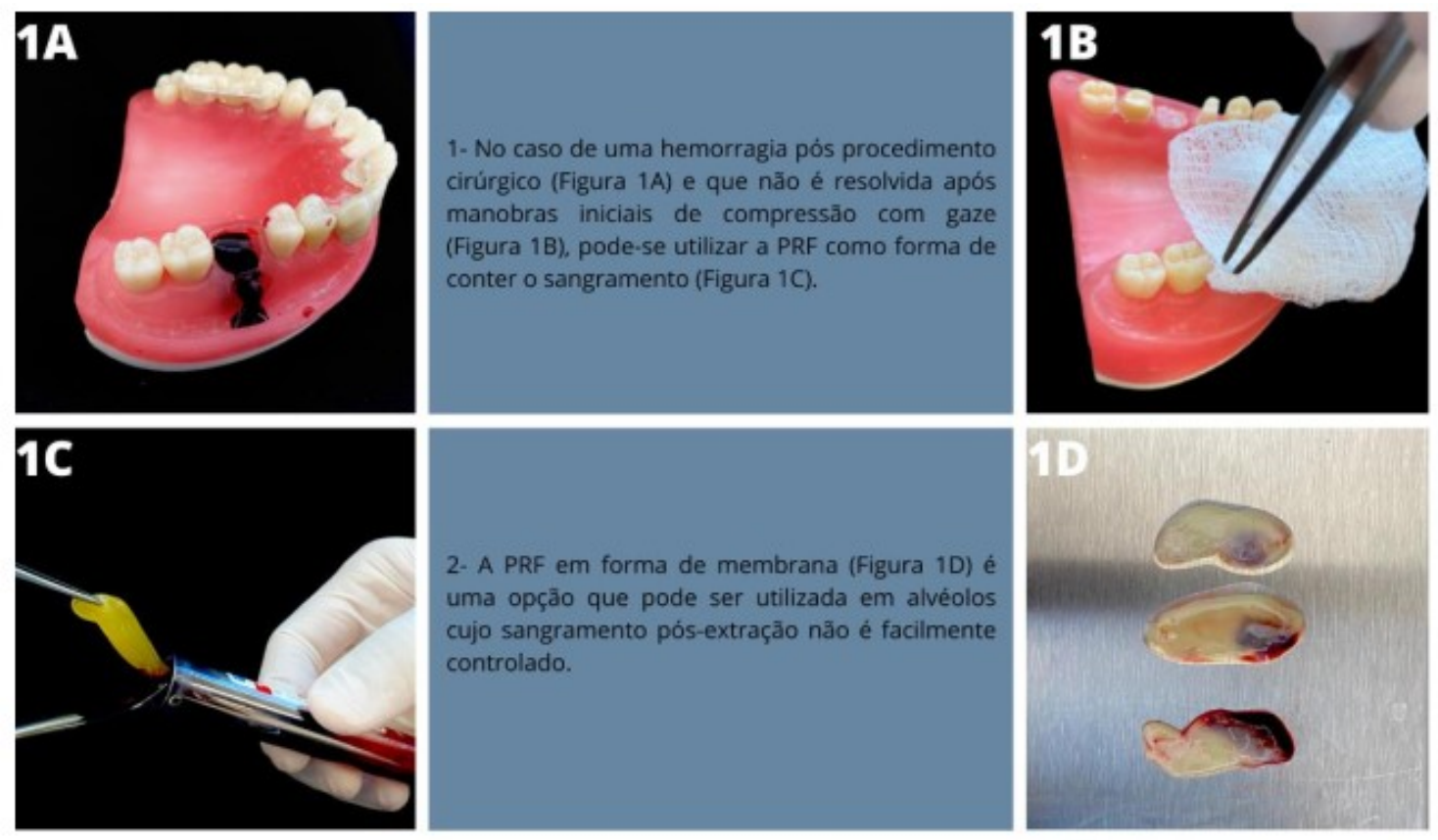

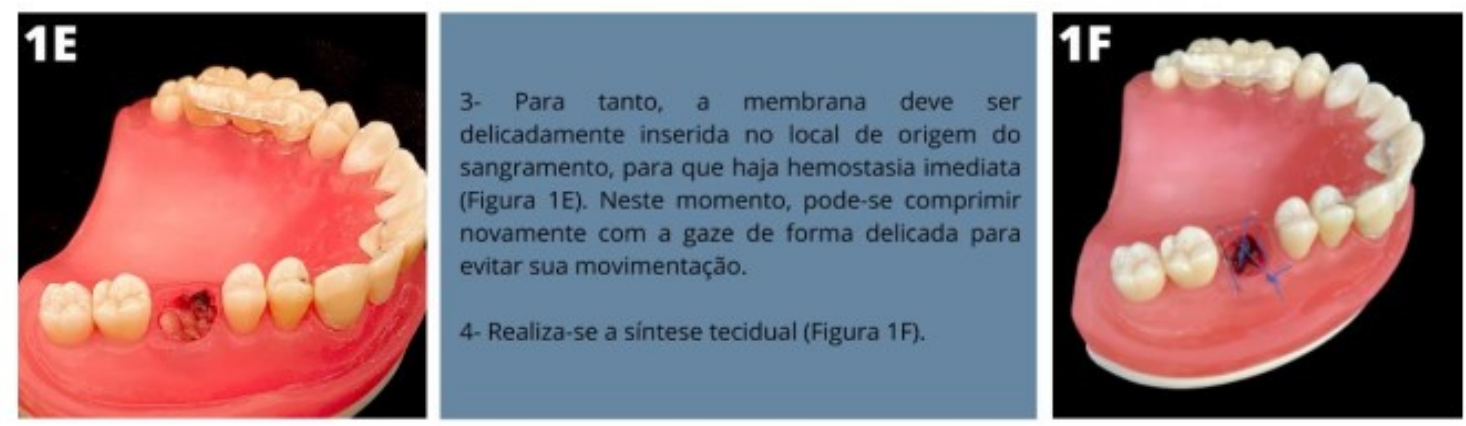

Figura 1: Instruções sobre o uso da PRF como hemostático.

\section{CONCLUSÃO}

A PRF é uma ótima técnica para a hemostasia do alvéolo dentário após exodontia, promovendo um melhor e mais rápido reparo das lesões nos casos em que a compressão com gaze não é suficiente para conter o sangramento. Além de ser de fácil reprodutibilidade, utilização e possuir um baixo custo.

\section{REFERÊNCIAS BIBLIOGRÁFICAS}

1. ALZAHRANI, Ahmed Abdullah; MURRIKY, Afraa; SHAFIK, Sami. Influence of platelet rich fibrin on post-extraction socket healing: A clinical and radiographic study. The Saudi dental journal, v. 29, n. 4, p. 149-155, 2017.

em: <https://www.sciencedirect.com/science/article/pii/S1013905217300536> Acesso em: 05 jul. 2021.

2. CARDAROPOLI, G .; ARAUJO, M .; LINDHE, J. Dinâmica da formação do tecido ósseo em sítios de extração dentária: um estudo experimental em cães. Journal of Clinical Periodontology , v. 30, n. 9, pág. 809-818, $2003 . \quad$ Disponível em: $<$ https://onlinelibrary.wiley.com/doi/abs/10.1034/j.1600051X.2003.00366. x> Acesso em: 05 jul. 2021. 
3. ARAÚJO, Mauricio G .; LINDHE, Jan. Alterações do rebordo dimensional após extração dentária.

4. Um estudo experimental no cão. Journal of Clinical Periodontology, v. 32 , n. 2, pág. 212-218, 2005. Disponível em: https://onlinelibrary.wiley.com/doi/abs/10.1111/j.1600051X.2005.00642.x Acesso em: 05 jul. 2021.

5. MOURÃO, C. F. A. B. et al. O uso da fibrina rica em plaquetas como biomaterial hemostático em complicações de exodontia dos terceiros molares. Revista do Colégio Brasileiro de Cirurgiões, v.3, p. 100-105, 2017. Disponível em: http://relatosdocbc.org.br/detalhes/110/o-uso-dafibrina-ricaem-plaquetas-como-biomaterial-hemostatico-emcomplicacoes-de-exodontia-dos-terceiros-molares Acesso em: 05 jul. 2021.

6. PAN, Jiayu et al. Effect of platelet-rich fibrin on alveolar ridge preservation: A systematic review. The Journal of the American Dental Association, v. 150, n. 9, p. 766-778, 2019. Disponível em: <https://pubmed.ncbi.nlm.nih.gov/31439204/> Acesso em: 05 jul. 2021.

7. DEL FABBRO, Massimo et al. Healing of postextraction sockets preserved with autologous platelet concentrates. A systematic review and meta-analysis. Journal of Oral and Maxillofacial Surgery,v. 75, n. 8, p. 1601-1615, 2017. Disponível em:<https://www.sciencedirect.com/science/article/abs/pii/S0278239117302185 $>$ Acesso em 05 jul.2021.

8. DEL FABBRO, M. et al. Autologous platelet concentrates to improve post extraction outcomes.Journal of Oral and Maxillofacial Surgery, v. 75, p. 16011615, 2017. 